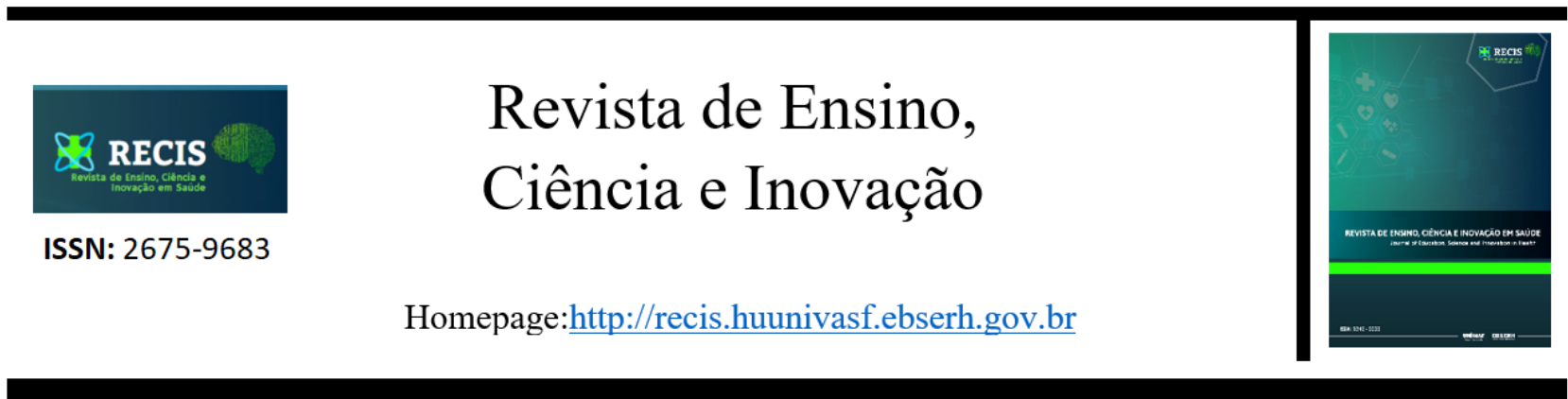

\title{
Perfil epidemiológico de acidentes envolvendo animais peçonhentos no Sertão do Estado de Pernambuco (2009 -2019)
}

\section{Epidemiological profile of accidents involving venomous animals in the Sertão of the State of Pernambuco (2009-2019)}

\author{
Carolline Xavier de Aguiar ${ }^{\mathbf{1}}$, Darllington Weslley Souza Gomes, ${ }^{\mathbf{1}}$ Drako de Amorim Souza ${ }^{\mathbf{1}}$, \\ Júlia Assucena Medrado de Castro', Lucas Braga dos Santos ${ }^{1}$, Mateus Antunes de Carvalho \\ Santos$^{1}$, Wanessa Nunes de Sousa ${ }^{1}$, Welisson Conrado Carvalho ${ }^{1}$, César Augusto da Silva ${ }^{2}$ \\ ${ }^{1}$ Discente, Colegiado de Medicina, Campus Petrolina. ${ }^{2}$ Docente, Colegiado de Medicina, Campus Petrolina. \\ Universidade Federal do Vale do São Francisco (UNIVASF).
}

Autor correspondente: cesar.silva@univasf.edu.br

Artigo recebido em 04/02/2021 e aceito em 05/04/2021

\begin{abstract}
RESUMO
Acidentes com animais peçonhentos apresentam alta incidência no Brasil e demonstram crescimento de ocorrências devido à persistência dos desmatamentos e à não utilização de materiais de proteção individual nas atividades do campo. Pertencentes à lista de doenças negligenciadas, sua subnotificação no país contribui para essa situação. Com o objetivo de sistematizar dados acerca dos acidentes com animais peçonhentos, foi realizado um estudo observacional, retrospectivo e descritivo dos casos desses acidentes confirmados e notificados na Macrorregião de Saúde do Vale do São Francisco e Araripe e Macrorregião de Saúde do Sertão, no Estado de Pernambuco. As informações foram coletadas pelo banco de dados do Departamento de Informática do Sistema Único de Saúde do Brasil. A análise dos dados mostrou que a incidência de notificação dos casos aumentou de 35,88 para 247,01/100.000 habitantes no período estudado; os animais que mais causam essa categoria de acidente são escorpiões (42,9\%), abelhas (29,3\%) e serpentes (16,3\%); sendo as serpentes mais envolvidas nesses episódios, as espécies dos gêneros Bothrops (86,8\%) e Crotalus (8,9\%); a maioria dos pacientes envolvidos evoluem para casos leves (78,8\%); além disso, o grau de escolaridade mais prevalente nos pacientes é de Ensino Fundamental I incompleto (12,45\%). Os dados aqui apresentados podem ser utilizados para orientar o desenvolvimento de campanhas de profilaxia e tratamentos adequados no país, auxiliando gestores de saúde e facilitando o trabalho das equipes da atenção básica em saúde.

Palavras-chave: Epidemiologia, Mordeduras e Picadas, Animais Venenosos, Doença Negligenciada.
\end{abstract}

\begin{abstract}
Accidents with venomous animals have a high incidence in Brazil and show an increase in occurrences due to the persistence of deforestation and the failure to use individual protection materials in field activities. Belonging to the list of neglected diseases, their underreporting in the country contributes to this situation. In order to systematically analyze the data about accidents with venomous animals, an observational, retrospective and descriptive study of confirmed and reported cases was carried out in the Health Macroregion of the Vale do São Francisco and Araripe and the Health Macroregion of Sertão, in the State of Pernambuco. The information was collected by the database of the Department of Informatics of the Brazilian Unified Health System. Data analysis showed that the incidence of notification of cases increased from 35.88 to $247.01 / 100.000$ inhabitants in the studied period; the animals that most cause this type of accident are scorpions $(42.9 \%)$, bees $(29.3 \%)$ and snakes $(16.3 \%)$; snake types most involved in these episodes are the species of the genera Bothrops $(86.8 \%)$ and Crotalus $(8.9 \%)$; most of the patients involved evolve to mild cases $(78.8 \%)$; in addition,
\end{abstract}

Aguiar CX, et al./Revista de Ensino, Ciência e Inovação em Saúde v.2 n.1(2021) p.27-36 
as to the level of education, the most prevalent in patients is incomplete Elementary Education (12.45\%). The data presented here can be used to guide the development of prophylaxis campaigns and appropriate treatments in the country, assisting health managers and facilitating the work of primary health care teams.

Keywords: Epidemiology, Bites and Stings, Poisonous Animals, Neglected Disease.

\section{INTRODUÇÃO}

Os animais peçonhentos são definidos como um grupo de animais que possuem glândulas especializadas em produzir veneno que se comunicam com o meio externo por meio de dentes ocos, ferrões ou aguilhões. ${ }^{1}$ Desses, cobras, escorpiões, aranhas e abelhas são os principais agentes de intoxicação de maior notoriedade clínica. Essa diversidade de espécies gera graves acidentes, que podem resultar em sequelas incapacitantes e até mesmo morte..$^{2,-3}$

Segundo a Organização Mundial da Saúde, acidentes com animais peçonhentos representam etiologia considerável de morbimortalidade em todo o mundo. ${ }^{4}$ Trata-se de um grande problema de saúde pública negligenciado, especialmente em países tropicais, o que motivou a Organização Mundial da Saúde (OMS) a incorporá-los na lista de Doenças Tropicais Negligenciadas (DTN) em 2009. . $-6^{-1}$

No Brasil, a variedade de espécies responsáveis por essa categoria de acidente se explica pela sua diversidade de biomas. ${ }^{7}$ Além disso, o desequilíbrio ecológico causado pelo homem ao longo dos anos, assim como o crescimento desordenado dos centros urbanos, têm sido alguns dos principais motivos da justaposição no uso do espaço entre o ser humano e esses animais, de tal forma que tal conjuntura aumenta a probabilidade da ocorrência destes eventos. ${ }^{2}$

Acidentes por animais peçonhentos se expressam como a segunda causa de intoxicação humana no país, sendo a primeira o uso de medicamentos. ${ }^{2,5}$ Essa circunstância se apresenta, inúmeras vezes, como Acidentes de Trabalho (AT) envolvendo, principalmente, trabalhadores do campo, floresta e águas, tornando-os mais vulneráveis a esse incidente. ${ }^{5}$

O Boletim Epidemiológico do Ministério da Saúde aponta que o perfil dos acometidos pelos acidentes envolvendo animais peçonhentos no Brasil é: do sexo masculino, na faixa etária produtiva e com baixa escolaridade. ${ }^{5}$ Esse dado corrobora com o perfil encontrado por diversos autores, envolvendo, sobretudo, escorpiões, serpentes e aranhas. ${ }^{1,8,9}$

Estes episódios passaram a ser agravos de notificação no Brasil a partir do ano de 1986, possibilitando subsídios para a identificação da realidade epidemiológica de cada região. ${ }^{1,10} \mathrm{Em}$ 1995 foi adotado o Sistema de Informações de Agravos de Notificação (SINAN) pela Coordenação Nacional de Controle de Zoonoses e Animais Peçonhentos (CNCZAP), de início com pouca adesão dos municípios, visto a inexistência de regulamentação oficial pelo Ministério da Saúde (MS), fato esse que despertou objeções dos Estados e Municípios ao novo sistema. Somente em 1998 o MS regulamentou, através de portaria, a obrigatoriedade de notificação dos agravos compulsórios pelos Municípios, Estados e Distrito Federal. Contudo, apesar dos resultados crescentes que demonstram uma melhoria na captação dos casos, os dados epidemiológicos do país ainda são inconsistentes devido às subnotificações. ${ }^{10,11}$

Dessa forma, embora a incidência de casos no Nordeste seja de 94/100.000 habitantes, a mais alta comparada às demais regiões do país (93,8/100.000 no Sul, 85,3/100.000 no Norte, $76,6 / 100.000$ no Sudeste e 59,3/100.000 habitantes no Centro-oeste) ainda pouco se sabe sobre o perfil clínico e epidemiológico desses acidentes nessa região. ${ }^{3,8}$ Nesse sentido, a importância do presente trabalho está em poder auxiliar no planejamento de intervenções preventivas e gestão de condutas frente a dimensão real dos problemas que envolvem este incidente, bem como, em contribuir com a proteção da população exposta. ${ }^{6,9,12}$

Nesse contexto, este estudo tem por objetivo analisar dados epidemiológicos a respeito de acidentes envolvendo animais peçonhentos registrados entre 2009 e 2019 na Macrorregião de Saúde do Vale do São Francisco e Araripe e na Macrorregião de Saúde do Sertão, no Estado de Pernambuco, com propósito de dispor de dados que possam contribuir com o planejamento de estratégias para a melhor condução destes eventos.

\section{MATERIAL E MÉTODOS}

Esse estudo é caracterizado como observacional, retrospectivo e descritivo dos casos confirmados e notificados de acidentes por animais peçonhentos na Macrorregião de Saúde do Vale do São Francisco e Araripe e na Macrorregião de Saúde do Sertão de Pernambuco, que incluem 60 municípios, ${ }^{13}$ e aproximadamente 1.863 .091 habitantes. ${ }^{14}$ Os dados foram obtidos no banco sobre Informação de Saúde do Ministério da Saúde 
- Departamento de Informática do Sistema Único de Saúde do Brasil (Datasus/MS), de acordo com município de residência, no período de 2009 a 2019, onde foram obtidas estimativas para número de residentes e casos de acidentes por animais peçonhentos.

Após a obtenção dos dados, foi calculado o coeficiente de incidência dos acidentes por animais peçonhentos, tendo como base o número de novos casos identificados nas Macrorregiões do estudo por período, multiplicado por 100.000 e dividido pela população notificada no período considerado. Foram comparados dados de incidência no Brasil, na região Nordeste e nos municípios de Petrolina e Serra Talhada, as duas últimas, sedes das duas macrorregiões estudadas. ${ }^{13}$ Foram também considerados para cálculos: valores absolutos e relativos de notificações, de incidência, de gravidade e evolução dos casos com relação a cada tipo de agente etiológico; valores absolutos e relativos de acidentes por categoria de serpente; e valores absolutos e relativos do número de notificações de acidentes por animais peçonhentos estratificado por nível de escolaridade. Os dados foram calculados considerando o período de 2009 a 2019 e a população notificada em cada uma dessas zonas.

Em epidemiologia, valores relativos são números resultantes de uma razão de coeficientes ou taxas, podendo ser interpretados como uma comparação em porcentagem de dois valores relativizados, enquanto os valores absolutos representam um indicador de saúde sem comparação a outro,,$^{15}$ como é o caso da incidência de acidentes por animais peçonhentos em uma certa região, num dado tempo e dada população.

Para analisar a discrepância no crescimento da incidência nos períodos de 2009 a $2016\left(\mathrm{P}_{1}\right)$ e 2017 a $2019\left(\mathrm{P}_{2}\right)$, foram calculadas as médias aritméticas das incidências para cada período ( $\mathrm{I}_{\mathrm{c}} \mathrm{P}_{1}$ e $\mathrm{I}_{\mathrm{c}} \mathrm{P}_{2}$, respectivamente) e estimadas as variações nessas médias em dois tipos: variações relativas $\left(\mathrm{V}_{\mathrm{R}}\right)$ e absolutas $\left(\mathrm{V}_{\mathrm{A}}\right)$. A primeira expressa em quantos por cento a média do segundo período (P2) aumentou, se comparada ao primeiro período (P1); enquanto que a segunda resulta no valor absoluto de aumento.

Seu cálculo se deu pelas seguintes expressões: $\mathrm{V}_{\mathrm{R}}=\left(\mathrm{I}_{\mathrm{c}} \mathrm{P}_{2}-\mathrm{I}_{\mathrm{c}} \mathrm{P}_{1}\right) / \mathrm{I}_{\mathrm{C}} \mathrm{P}_{1} * 100 \% ; \mathrm{V}_{\mathrm{A}}=$ $\mathrm{I}_{c} \mathrm{P}_{2}-\mathrm{I}_{\mathrm{C}} \mathrm{P}_{1}$. Essas variações foram estimadas para cada agente etiológico com objetivo de identificar os principais responsáveis pelo nível elevado de notificações em $\mathrm{P}_{2}$.

Os dados foram coletados através de sistema de informações de domínio público, com acesso irrestrito, sem obtenção de informação sigilosa ou individual, obedecendo aos princípios éticos da Resolução 466/2012 ${ }^{16}$ e da Resolução 510/2016 ${ }^{17}$ do Conselho Nacional de Saúde, de forma que é dispensada a necessidade de análise do trabalho pelo Comitê de Ética em Pesquisa.

\section{RESULTADOS}

Os dados disponíveis no sistema Datasus/MS mostram que a Macrorregião de Saúde do Vale do São Francisco e Araripe e a Macrorregião de Saúde do Sertão de Pernambuco, tendo como cidades sede Petrolina e Serra Talhada, respectivamente, registraram o total de 21.358 casos de acidentes com animais peçonhentos no período de 2009 a 2019. Em 2019, foi notificado o maior número de casos $(\mathrm{n}=4.602)$, com coeficiente de incidência de 247/100.000 habitantes. Além disso, a partir de 2017 as duas Macrorregiões passaram a demonstrar maior taxa de notificação $(135,60 / 100.000$ habitantes) que a região Nordeste $(134,65 / 100.000$ habitantes) e que o Brasil na totalidade (107,99/100.000 habitantes). Esse crescimento foi suficiente para que as macrorregiões pernambucanas superassem a região Nordeste em 49,00\% e o Brasil em 95,36\% no ano de 2019 (Figura 1).

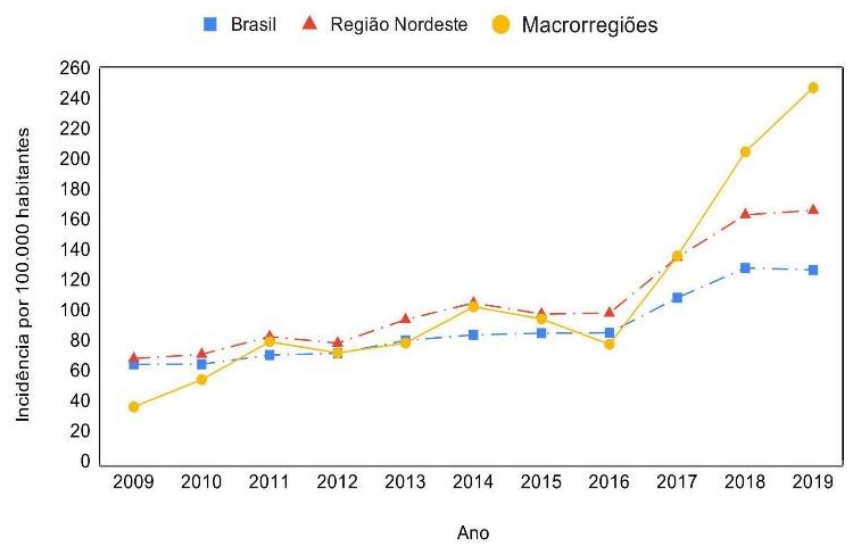

Figura 1. Comparativo entre as taxas de Incidências (por 100.000 habitantes) de acidentes por animais peçonhentos na Macrorregião de Saúde do Vale do São Francisco e Araripe, na Macrorregião de Saúde do Sertão de Pernambuco, no Brasil e na região Nordeste, no período de 20092019.

Dentre os acidentes por tipo de animais peçonhentos, durante o período estudado, os dados disponíveis mostram que a maior parte deles ocorreu por escorpiões $(42,9 \%)$, abelhas $(29,3 \%)$ e serpentes $(16,3 \%)$, seguidos por aranhas $(3,5 \%)$ e lagartas $(0,7 \%)$. A causa do acidente não foi identificada em 393 casos $(1,8 \%)$ e $1.116(5,2 \%)$ ocorreram por outros animais peçonhentos.

Ao se investigar a origem do aumento de notificações a partir de 2017, foi possível notar que, 
Revista de Ensino, Ciência e Inovação em Saúde v.2, n.1 (2021) 27-36

embora todos os acidentes tenham apresentado aumento no período de 2009 a 2016, houve exacerbação no valor absoluto da média de notificações de acidentes por escorpiões e abelhas a partir de 2017 (Tabela 1). Esses dois agentes são, portanto, os principais responsáveis pelo pico na incidência.

Quanto à gravidade dos acidentes, os dados apresentados na tabela 2 , mostram que serpentes, escorpiões e abelhas são responsáveis pelos maiores números de casos graves $(81,25 \%$, $8,26 \%$ e $6,45 \%$ respectivamente), mesma tendência observada quando se refere à evolução dos casos, onde se destacaram, pela letalidade, notadamente as serpentes $(0,46 \%)$ e as abelhas $(0,24 \%)$; aranhas apresentaram taxa de letalidade maior $(0,13 \%)$ que escorpiões $(0,09 \%)$.

Tabela 1: Incidência de acidentes para cada 100.000 habitantes em relação ao tipo de agente etiológico, nas macrorregiões do estudo, nos períodos 2009 a 2016 e 2017 a 2019

\begin{tabular}{ccccc}
\hline \multirow{2}{*}{$\begin{array}{c}\text { Agentes } \\
\text { etiológicos }\end{array}$} & $\begin{array}{c}\text { Incidência média e IC* (95\%) } \\
\text { para intervalo em anos }\end{array}$ & \multicolumn{2}{c}{ Variações das médias } \\
& $\mathbf{2 0 0 9}|-| \mathbf{2 0 1 6}$ & $\mathbf{2 0 1 7}|-| \mathbf{2 0 1 9}$ & Relativas (\%) & Absolutas \\
\hline Ign**/branco & $1,68 \pm 0,39$ & $2,77 \pm 0,56$ & $+64,90 \%$ & $+1,09$ \\
Serpente & $17,50 \pm 3,96$ & $18,69 \pm 2,15$ & $+6,77 \%$ & $+1,18$ \\
Aranha & $2,61 \pm 0,66$ & $6,99 \pm 1,62$ & $+167,73 \%$ & $+4,38$ \\
Escorpião & $27,43 \pm 8,69$ & $95,02 \pm 18,03$ & $+246,37 \%$ & $+67,58$ \\
Lagarta & $0,36 \pm 0,16$ & $1,82 \pm 0,41$ & $+399,15 \%$ & $+1,45$ \\
Abelha & $20,98 \pm 8,21$ & $58,96 \pm 13,83$ & $+181,06 \%$ & $+37,98$ \\
Outros & $3,38 \pm 1,15$ & $11,46 \pm 3,10$ & $+239,14 \%$ & $+8,08$ \\
\hline
\end{tabular}

*IC (95\%): intervalo de confiança de 95\% para uma distribuição normal.

**Ign: ignorado.

Tabela 2: Gravidade e evolução dos casos de acidentes nas macrorregiões de estudo, no período de 2009 a 2019, em relação a cada tipo de agente etiológico

Gravidade do caso

Tipo de Acidente

\begin{tabular}{ccccccc} 
Tipo de Acidente & Leve & Moderado & Grave & Cura & $\begin{array}{c}\text { Óbito pelo } \\
\text { agravo } \\
\text { notificado }\end{array}$ & Letalidade \\
\hline Ign*/Branco & 258 & 42 & 7 & 319 & 0 & $0,00 \%$ \\
Serpente & 1.631 & 1.066 & 403 & 2.993 & 16 & $0,46 \%$ \\
Aranha & 577 & 137 & 9 & 695 & 1 & $0,13 \%$ \\
Escorpião & 8.025 & 719 & 41 & 8.655 & 8 & $0,09 \%$ \\
Lagarta & 129 & 17 & - & 141 & 0 & $0,00 \%$ \\
Abelha & 5.244 & 705 & 32 & 5.996 & 15 & $0,24 \%$ \\
Outros & 964 & 111 & 4 & 1.082 & 0 & $0,00 \%$ \\
\hline
\end{tabular}

*Ign: ignorado.

A análise dos municípios de Petrolina e Serra Talhada, sedes das duas macrorregiões deste estudo, ${ }^{13}$ mostra que o total de casos notificados na primeira $(\mathrm{n}=2.708)$ corresponde a $12,68 \%$ do total de casos, enquanto que na segunda $(n=1.673)$ corresponde a $7,83 \%$. Entretanto, ao analisar a incidência dos acidentes na cidade de Serra Talhada, identifica-se uma taxa de incidência maior nesta cidade quando comparada à taxa de incidência nas macrorregiões como um todo ou na 
cidade de Petrolina isoladamente (Figura 2). Isso se deve ao fato de Serra Talhada concentrar um grande número de notificações e ter apresentado uma população média reduzida no período de 2009 a $2019(n=83.458)$ em comparação às populações de Petrolina $(n=321.164)$ e das macrorregiões somadas $(\mathrm{n}=1.788 .941) .{ }^{18}$

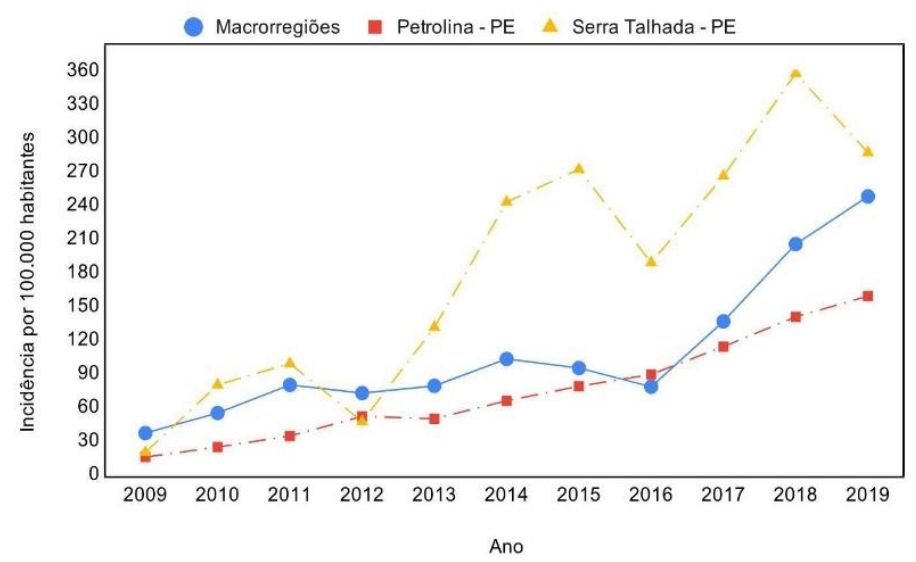

Figura 2. Taxa de Incidência de acidentes por animais peçonhentos em Petrolina/PE, Serra Talhada/PE e nas macrorregiões estudadas, no período de 2009-2019

A análise dos acidentes envolvendo apenas as serpentes mostrou que o gênero Bothrops foi responsável pelo maior número de casos em todos os anos pesquisados neste estudo $(n=1881)$, correspondendo a $53,74 \%$ do total de casos; os demais foram registrados como sendo causados pelos gêneros Crotalus $(\mathrm{n}=392)$, Micrurus $(\mathrm{n}=96)$, Lachesis $(\mathrm{n}=12)$ e não peçonhentos $(\mathrm{n}=287)$. No total, o número de notificações ignoradas ou em branco $(n=832)$ correspondeu a $23,77 \%$ do total de casos por serpentes no período $(n=3500)$, com máximo de 33,78\% em 2015 (100/296) e mínimo de 17,14\% em 2009 (60/350).

Quando analisadas as notificações de acidentes por animais peçonhentos em relação ao sexo, observou-se maior prevalência média em indivíduos do sexo masculino $(55,59 \%)$ durante todo o período (Figura 3). Em 2009 foram notificados 613 casos, sendo 66,88\% deles em homens; em 2019, foram notificados 4.602 casos, dos quais $54,28 \%$ em homens. É interessante notar que, embora o número de casos tende a crescer $\left(\mathrm{R}^{2}=0,754\right)$ com o passar dos anos, a proporção entre o sexo masculino e o feminino, antes com diferença acentuada, tende a alcançar os $50 \%\left(\mathrm{R}^{2}\right.$ $=0,603$ ).

Ao investigar a relação entre sexo e tipos específicos de acidentes, notou-se que a tendência geral em relação ao sexo masculino predominou em todos os casos, exceto nos acidentes por escorpião $(56,40 \%$ para o sexo feminino). Ficou evidente, também, a majoritária porção de acidentes no sexo masculino por serpentes $(74,94 \%)$ e abelhas $(63,34 \%)$.

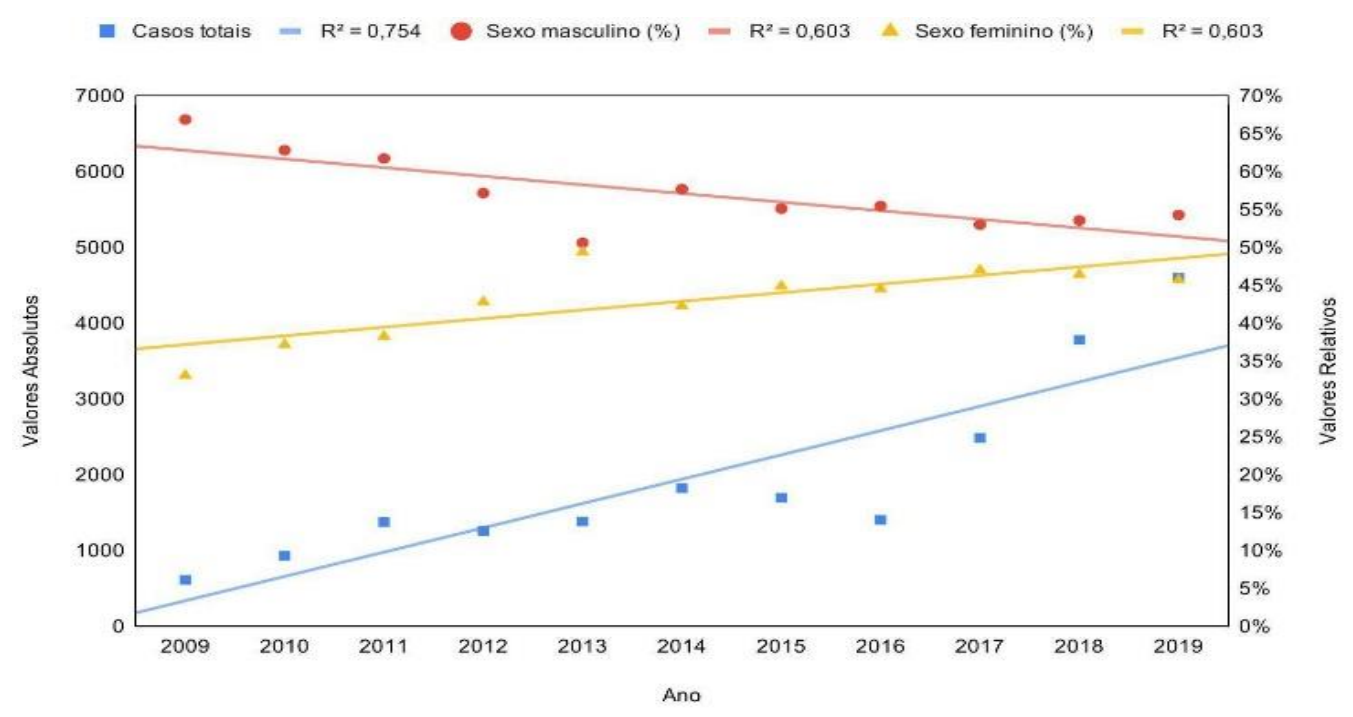

Figura 3. Casos de acidentes por animais peçonhentos confirmados nas Macrorregiões de Saúde do Vale do São Francisco e Araripe e Macrorregião de Saúde do Sertão de Pernambuco por ano, no período de 2009-2019, de acordo com o sexo dos envolvidos

Vários trabalhos mostram que o grau de instrução da população está diretamente relacionado aos índices de infecção por DTN. ${ }^{19,20}$ Neste sentido, o presente estudo buscou ainda traçar a relação entre esses parâmetros na área de 
trabalho e ao serem analisadas as notificações de acidentes de acordo com o grau de escolaridade do indivíduo, observou-se que, na maioria dos casos, esse parâmetro foi ignorado, não preenchido ou preenchido como "não se aplica", dado que corresponde a $44,51 \%$ do total de casos notificados (Tabela 4). Este preenchimento incompleto ou inadequado das informações de notificação claramente reflete uma realidade enfrentada por muitos pesquisadores que utilizam o sistema Datasus/MS como fonte de pesquisa. É importante destacar que além de não representar a situação real dos municípios frente às DTN, tal fato põe em risco o planejamento e execução de estratégias educativas e preventivas, tanto para as DTN quanto para os acidentes envolvendo animais peçonhentos.

Os dados mostram ainda que pessoas com ensino fundamental I e II incompletos foram as mais acometidas $(24,41 \%)$, enquanto que, aquelas com ensino superior completo ou incompleto, foram as menos acometidos $(2,93 \%)$ por acidentes com animais peçonhentos (Tabela 4). Esse dado corrobora que o nível de escolaridade está diretamente relacionado ao aumento do número de ocorrências, daí a importância do preenchimento correto das informações de notificação, que mostrarão a realidade de cada local e permitirão o planejamento e execução de medidas educativas que possam mitigar os problemas de saúde no país.

Tabela 4: Número de notificações de acidentes nas macrorregiões de estudo, estratificado por nível de escolaridade, no período de 2009 até 2019

\begin{tabular}{ccc}
\hline Grau de escolaridade (2009 a 2019) & Número de Notificações & $\begin{array}{c}\text { \% do total } \\
\text { de dados }\end{array}$ \\
\hline Ignorados/Não preenchido & 6.882 & $32,22 \%$ \\
Analfabeto & 616 & $2,88 \%$ \\
$1^{\mathrm{a}}$ a $4^{\mathrm{a}}$ série (Fundamental I incompleto) & 2.660 & $12,45 \%$ \\
Fundamental I completo & 895 & $4,19 \%$ \\
$5^{\mathrm{a}}$ a $8^{\mathrm{a}}$ série (Fundamental II incompleto) & 2.554 & $11,96 \%$ \\
Fundamental II completo & 863 & $4,04 \%$ \\
Ensino médio incompleto & 1.089 & $5,10 \%$ \\
Ensino médio completo & 2.551 & $11,94 \%$ \\
Educação superior incompleta & 224 & $1,05 \%$ \\
Educação superior completa & 402 & $1,88 \%$ \\
Não se aplica & 2.625 & $12,29 \%$ \\
TOTAL & $\mathbf{2 1 . 3 6 1}$ & $\mathbf{1 0 0 , 0 0 \%}$ \\
\hline
\end{tabular}

\section{DISCUSSÃO}

A análise dos dados apresentados mostrou que, dos 21.358 casos de acidentes por animais peçonhentos ocorridos no período de 2009 a 2019, nas Macrorregiões estudadas, mais de $50 \%$ ocorreram nos anos de 2017 a 2019. Comparativamente, na região Nordeste como todo, o período de 2017 a 2019 registrou cerca de um terço desses acidentes. ${ }^{21}$ Além disso, nas macrorregiões deste estudo, foi observado aumento de $650,73 \%$ no número de notificações de acidentes do ano de 2009 para o de 2019 , enquanto isso, a região Nordeste, para o mesmo período, apresentou aumento de $159,50 \% .^{21}$ Tais dados, aliados à localização geográfica da Universidade Federal do Vale do São Francisco, justificam a escolha das macrorregiões de estudo.

Esse aumento no número de casos, do início para o final do período de estudado, notadamente nos três últimos anos, talvez esteja relacionado a fatores como aumento da taxa de 
urbanização, desmatamento de mata nativa, trabalhos relacionados ao campo ou ainda por ausência de políticas públicas e programas mais eficientes no combate a esses agravos. Ademais a maior procura por serviços de saúde, aumento geral do número de habitantes e melhoria no sistema de coleta de dados também podem ser vistos como contribuidores. ${ }^{6,21}$

No Brasil, os casos de acidentes por animais venenosos são estudados há mais de um século e a diversidade territorial traz dificuldades para a avaliação, principalmente quando se utilizam dados de locais específicos com o objetivo de generalizar resultados. ${ }^{6}$ Essa dificuldade, felizmente, está sendo reduzida desde a implantação do SINAN.

A utilização do SINAN permitiu traçar algumas tendências no país no período de 2001 a 2012. Nesse período, a incidência média para acidentes com serpentes, aranhas, escorpiões, abelhas e lagartas, apresentava-se crescente linearmente, algo em torno de 55 ataques $/ 100.000$ habitantes. Os principais animais responsáveis pela taxa de letalidade $(<0,50 \%)$ foram as serpentes $(0,43 \%)$ e as abelhas $(0,33 \%)$. O Nordeste brasileiro se destacou por ser a região com maior incidência de acidentes por escorpiões, sendo o terceiro em acidentes por serpentes (abaixo do norte e centro-oeste). ${ }^{6}$ Este comportamento de aumento linear é condizente com os dados deste trabalho e permaneceu linear até o ano de 2016, onde pôde ser visto um aumento na incidência dos acidentes a partir de 2017 ao nível Nacional, Nordeste e nas macrorregiões estudadas (Figura 1).

Nos resultados pôde-se verificar que picadas por escorpiões representaram $42,97 \%$ dos acidentes por animais peçonhentos nas macrorregiões estudadas, além de serem os agentes que mais contribuíram para o aumento da incidência de acidentes nessa área (Tabela 1). Estes casos também são importantes pela sua potencial gravidade, principalmente em crianças picadas pelo Tityus serrulatus, ${ }^{12}$ que possuem grande capacidade de adaptação e reprodução partenogenética, contribuindo para sua ampla distribuição. ${ }^{22}$ Ademais, o avanço das cidades, falta de saneamento básico e as precárias construções sociais proporcionam a esses animais um ambiente com ausência de predadores naturais, alimento de fácil acesso e abrigo. ${ }^{21}$

Também causa preocupação o aumento de 181,06\% na incidência de picadas de abelhas neste estudo (Tabela 1), isto porque estas são uma das responsáveis pelas maiores taxas de letalidade, tanto no país quanto nas macrorregiões estudadas (Tabela 2). Esse fenômeno pode ser explicado pela capacidade de certas espécies (dentre elas a abelha africanizada, considerada agressiva) de nidificar em estruturas da cidade ou do campo, conforme o homem adentra o seu habitat silvestre. ${ }^{23}$ Ao estudar situação parecida em Campina Grande/PB, os autores firmaram hipótese similar, argumentando que a disponibilidade de recursos como água, somada à ação antropogênica sobre seus habitats, são fatores que podem explicar o aumento na notificação de picadas por abelhas. ${ }^{24}$

Além disso, estudos mostram um aumento de $96,26 \%$ na taxa de produção de mel no Brasil no período de 1999 a 2009, com destaque para a região Nordeste que apresentou crescimento de 435,36\% no mesmo período. Entre os dez municípios com atividade apícola de relevância no país em 2009, oito deles são da região Nordeste, estando o município de Araripina, no Sertão de Pernambuco, ocupando o segundo lugar no ranking. As mesorregiões do Sertão e São Francisco apresentaram uma evolução da produção com variações de $3.668 \%$ e $1.740 \%$, respectivamente. ${ }^{25}$ Nas últimas décadas, tem havido um esforço por parte de órgãos estaduais e do Governo Federal incentivando a prática da apicultura familiar em Pernambuco através de políticas de inserção e expansão dessa atividade no Estado. ${ }^{26}$ Esse fato pode estar relacionado, também, ao aumento dos acidentes por picadas de abelhas nas macrorregiões estudadas.

Os acidentes envolvendo serpentes foram a terceira maior causa de notificação na área deste estudo, além de serem os únicos agentes que não sofreram grandes alterações em suas incidências no período estudado (Tabela 2). Nesse contexto, predominou acidentes por botrópicos $(53,74 \%)$, seguido por serpentes dos gêneros Crotalus $(11,20 \%)$, serpentes não peçonhentas $(8,2 \%)$, Micrurus $(2,74 \%)$ e Lachesis. Em estudo anterior, realizado no período de 2003 a 2012, no Brasil, foram notificados $86,8 \%$ de acidentes por Bothrops e $8,9 \%$ por Crotalus. ${ }^{7}$ Logo, o desfecho da presente análise corrobora com os resultados obtidos por outros autores, provavelmente refletindo uma situação nacional. ${ }^{12,27}$

A superioridade de acidentes por Bothrops pode estar relacionada à diversidade do gênero, que possui cerca de 30 espécies, elevada distribuição nos ecossistemas brasileiros, além de comportamento agressivo que, associados à sua ambientação nos meios antropizados, geram tais resultados. Por sua vez, as espécies do gênero Crotalus demonstram comportamento de menor agressividade, sendo encontradas em áreas mais abertas. Os acidentes com o gênero Micrurus, apresentam-se como raros devido, especialmente, à sua coloração e dentição. Picadas por indivíduos do gênero Lachesis representaram aqui apenas $0,34 \%$ 
do total de acidentes ofídicos, corroborando com outros trabalhos e ainda pelo fato de existirem poucos casos relatados na literatura. A causa atribuída para a escassez de informações sobre esses acidentes é a localidade na qual ocorrem, que são áreas florestais onde a densidade populacional é baixa e o sistema de notificação não é tão eficiente. ${ }^{7,12,27,28}$

Ainda, quanto ao tipo de serpente, destacase a quantidade de notificações assinaladas como ignoradas ou em branco, que representam $23,77 \%$ destes casos. A identificação dos acidentes de acordo com o gênero das serpentes é importante por auxiliar na indicação mais precisa do antiveneno a ser administrado e da conduta a ser tomada, além de viabilizar o reconhecimento das espécies de relevância médica em âmbito regional. ${ }^{27}$ Neste sentido, os dados do presente trabalho serão úteis porque poderão auxiliar os trabalhos das equipes da atenção básica em saúde.

Neste estudo, os acidentes por aranhas foram responsáveis por 3,5\% das notificações e 1 óbito, demonstrando baixa taxa de letalidade $(0,13 \%)$ (Tabela 1). Tal episódio está mais associado a acidentes domiciliares por meio do manuseio de resíduos de construção, lixo, ou até mesmo de calçados sem devida precaução. ${ }^{5,12}$

A análise do número de acidentes por animais peçonhentos que evoluiu para óbito, no período investigado, mostrou maior taxa de mortalidade no ano de $2011 \quad(0,469 / 100.000$ habitantes, 8 óbitos), enquanto a menor taxa de mortalidade ocorreu em $2012(0,058 / 100.000$ habitantes, 1 óbito). Importante destacar que a fonte de dados usada neste trabalho não dispõe de informações para este parâmetro no ano de 2018. Ressalta-se ainda que o programa Mais Médicos, implementado em 2013, permitindo maior acesso da população à saúde principalmente no interior dos Estados, talvez tenha contribuído com a redução no número de óbitos nos anos subsequentes. ${ }^{29}$

A relação entre os acidentes e o sexo dos pacientes, deste estudo, mostra prevalência de homens em todo período analisado (Figura 3), perfil epidemiológico que corrobora com outros estudos no Brasil, sendo explicado pelo predomínio de homens expostos em atividades laborais, especialmente nas atividades agrícolas rurais, ${ }^{27,28,30}$ onde tais acidentes representam um grande problema de saúde ocupacional. ${ }^{28} \mathrm{~A}$ predominância do sexo feminino em relação aos acidentes por escorpião pode estar relacionada ao tempo de exposição desse grupo aos ambientes e atividades domésticas em que entulhos, caixas de esgoto, frestas em paredes, podem se tornar abrigos para esses animais, dessa forma, tornando-as mais suscetíveis a esses eventos. ${ }^{31}$

Embora a quantidade de casos notificados tenha aumentado em cerca de $650,73 \%$, nas macrorregiões estudadas, a proporção da prevalência de acordo com o sexo diminuiu com o passar dos anos.

O presente estudo mostra ainda que parece haver uma relação inversamente proporcional entre o nível de escolaridade e o número de casos de acidentes (Tabela 4). Dado semelhante já foi observado na literatura. ${ }^{32}$ possivelmente porque existe uma relação direta entre grau de instrução e entendimento das medidas de prevenção. Assim, quanto maior o nível de informação de um indivíduo, melhores serão suas estratégias de prevenção. Contudo, essa afirmação não pode ser conclusiva, ao menos para a área estudada, visto que, de acordo com os resultados apresentados há um grande número de notificações onde $\mathrm{o}$ parâmetro escolaridade do paciente não foi adequadamente preenchido $(44,51 \%)$ (Tabela 4). Uma das justificativas possíveis é que a área de preenchimento na notificação referente a essa informação é um campo essencial, não obrigatório. Dessa forma, o profissional de saúde responsável por preencher a ficha de notificação pode deixar de registrá-lo, mesmo que seja necessário à investigação do caso ou ao cálculo do indicador epidemiológico ou operacional. ${ }^{33}$ Vale ainda destacar que em 12,29\% das notificações, o grau de escolaridade foi dado como "não se aplica", o que pode acontecer no caso de crianças em idade não escolar, por exemplo, cuja informação não teria aplicabilidade. Contudo, essa informação não pôde ser checada no momento da coleta de dados pelos pesquisadores.

Diante do exposto, se faz necessário prudência na interpretação dos dados e na construção de conclusões. Isto porque foram utilizados dados secundários de banco público, os quais estão sujeitos à subnotificação e ao preenchimento incorreto ou insatisfatório, podendo não representar a realidade sobre os acidentes por animais peçonhentos na área do estudo. Além disso, não se pode definir relações de causaconsequência ou associações, mas somente a descrição dos dados expostos devido ao tipo de estudo.

\section{CONCLUSÃO}

No período de 2009 a 2019, as macrorregiões estudadas apresentaram crescimento na incidência de notificações para acidentes com animais peçonhentos. Este crescimento foi similar na região Nordeste e no 
Brasil, além disso, desde 2017 a área investigada mostrou maior incidência de casos. $\mathrm{O}$ aumento na notificação de acidentes por escorpiões e abelhas é o principal responsável por essa elevação na incidência, sendo esses dois, os principais agentes etiológicos identificados nas notificações, principalmente, a partir de 2017. A maioria dos acidentes por serpentes está relacionada ao gênero Bothrops, além disso, as serpentes são as principais responsáveis pelos casos graves e mais letais nas macrorregiões. Por fim, as vítimas dos acidentes com animais peçonhentos neste estudo são, na maioria, homens de baixa escolaridade.

\section{REFERÊNCIAS}

1. Gonçalves CWB, Pinto Neto AB, Gomes DLF, et al. Acidentes com animais peçonhentos em um estado do norte do Brasil. SciGen. 2020;1(3):3743. Disponível em: http://www.scientiageneralis.com.br/index.php/S G/article/view/v1n3a3

2. Machado C. Um panorama dos acidentes por animais peçonhentos no Brasil. J Health NPEPS. 2016;1(1):1-3. Disponível em: https://periodicos.unemat.br/index.php/jhnpeps/art icle/view/1555

3. Tavares AV, Araújo KAM, Marques MRV, et al. Epidemiology of the injury with venomous animals in the state of Rio Grande do Norte, Northeast of Brazil. Ciênc. saúde coletiva. 2020;25:1967-1978. https://doi.org/10.1590/1413-

81232020255.16572018

4. World Health Organization (WHO). Rabies and envenomings. A neglected public health issue. Report of a consultative meeting. Geneva: World Health Organization; 2007. 38 p. Disponível em: https://apps.who.int/iris/bitstream/handle/10665/4 3858/9789241563482_eng.pdf?sequence $=1 \&$ isAll owed $=\mathrm{y}$

5. Secretaria de Vigilância em Saúde. Acidentes de trabalho por animais peçonhentos entre trabalhadores do campo, floresta e águas, Brasil 2007 a 2017. Boletim Epidemiológico. 2019;50:1$14 . \quad$ Disponível em: https://portalarquivos2.saude.gov.br/images/pdf/2 019/marco/29/2018-059.pdf

6. Chippaux JP. Epidemiology of envenomations by terrestrial venomous animals in Brazil based on case reporting: from obvious facts to contingencies. J. Venom. Anim. Toxins incl. Trop. Dis. 2015;21: 1-17. https://doi.org/10.1186/s40409-015-0011-1

7. Matos RR, Ignotti E. Incidência de acidentes ofídicos por gêneros de serpentes nos biomas brasileiros. Ciênc. saúde coletiva. 2020;25:2837-
2846.

81232020257.31462018

https://doi.org/10.1590/1413-

8. Costa MMR, Costa ES, Vilaça DHV, et al. Acidentes ofídicos: perfil epidemiológico na mesorregião do sertão Pernambucano, Brasil. Braz. J. Hea. Rev. 2018;1(1):245-251. Disponível em: https://www.brazilianjournals.com/index.php/BJH R/article/view/676

9. Silva AM, Bernarde PS, Abreu LC. Accidents with poisonous animals in Brazil by age and sex. J. Hum. Growth Dev. 2015;25(1):54-62. https://doi.org/10.7322/jhgd.96768

10. Bochner R, Struchiner CJ. Acidentes por animais peçonhentos e sistemas nacionais de informação. Cad. Saúde Pública. 2002;18(3): 735746. 311X2002000300017

11. Laguardia J, Domingues CMA, Carvalho C, et al. Sistema de informação de agravos de notificação em saúde (Sinan): desafios no desenvolvimento de um sistema de informação em saúde. Epidemiol. Serv. Saúde. 2004;13(3):135146. http://dx.doi.org/10.5123/S167949742004000300002

12. Oliveira ATAL, Sousa AFPB, Alcantara ICL, et al. Acidentes com animais peçonhentos no Brasil: revisão de literatura. Revinter. 2018;11(3):119-136.

http://dx.doi.org/10.22280/revintervol11ed3.389 13. Secretaria de Saúde do Estado de Pernambuco. Plano Diretor de Regionalização. Pernambuco, 2011. 2011. Disponível em: http://portal.saude.pe.gov.br/sites/portal.saude.pe. gov.br/files/pdrconass-

versao_final1.doc_ao_conass_em_jan_2012.pdf 14. Instituto Brasileiro de Geografia e Estatística (IBGE). Cidades e Estados. 2019. Disponível em: https://www.ibge.gov.br/cidades-e-estados/pe.htm 15. Merchán-Hamann E, Tauil PL, Costa MP. Terminologia das medidas e indicadores em epidemiologia: Subsídios para uma possível padronização da nomenclatura. Inf Epidemiol Sus. 2000;9(4). http://dx.doi.org/10.5123/S010416732000000400006

16. Brasil. Ministério da Saúde. Conselho Nacional de Saúde. Resolução no 466 , de 12 de dezembro de 2012. Trata de pesquisas em seres humanos e atualiza a resolução 196. Diário Oficial da União. Brasília (DF), 2012 dez 12.

17. Brasil. Ministério da Saúde. Conselho Nacional de Saúde. Resolução no ${ }^{\circ}$ 510, de 7 de Abril de 2016. Trata de diretrizes éticas específicas para as ciências humanas e sociais (CHS). Diário Oficial da União. Brasília(DF), 2016 abr 7.

18. Ministério da Saúde (BR). Sistema de Informação de Agravos de Notificação (Sinan). População residente - estudo de estimativas 
Revista de Ensino, Ciência e Inovação em Saúde v.2, n.1 (2021) 27-36

populacionais por município. 2020. Disponível em: http://tabnet.datasus.gov.br/cgi/deftohtm.exe?pops vs/cnv/popbr.de

19. Reis ACSM, Borges DPL, D’Ávila VGFC, et al. O cenário de políticas públicas do Brasil diante do quadro das doenças negligenciadas. Saúde \& Ciência em Ação. 2016;3(1):1-9. Disponível em: https://revistas.unifan.edu.br/index.php/RevistaIC S/article/viewFile/237/179

20. Rosário MS, Oliveira ML, Lima CA, et al. Doenças tropicais negligenciadas: caracterização dos indivíduos afetados e sua distribuição espacial. RBPS. 2017;19(3):118-127. https://doi.org/10.21722/rbps.v19i3.19574

21. Gonçalves JE, Medeiros SMFRS, Cavalcanti IDL, et al. Accidents caused by venomous animals: an analysis of the epidemiological profile in the Northeast region of Brazil in the period from 2010 to 2019. RSD. 2020;9(10):e4679108843. https://doi.org/10.33448/rsd-v9i10.8843

22. Sousa VA, Silva TTD, Sousa FDA, et al. Escorpiões de importância médica no estado da Paraíba, Brasil. Evidência. 2020;20(1):57-68. https://doi.org/10.18593/eba.24714

23. Santos AMM, Mendes EC. Abelha africanizada (apis mellifera 1.) em áreas urbanas no Brasil: necessidade de monitoramento de risco de acidentes. Sustinere. 2016;4(1). http://dx.doi.org/10.12957/sustinere.2016.24635

24. Linard ATS, Barros RM, Sousa JA, et al. Epidemiology of bee stings in Campina Grande, Paraíba state, Northeastern Brazil. J. Venom. Anim. Toxins incl. Trop. Dis. 2014;20:13. https://doi.org/10.1186/1678-9199-20-13

25. Serviço Brasileiro de Apoio às Micro e Pequenas Empresas (SEBRAE). Boletim setorial do agronegócio. Apicultura. 2011. Disponível em: https://www.sebrae.com.br/Sebrae/Portal\%20Sebr ae/Anexos/boletim-apicultura.pdf

26. Gomes RVRS, Miranda ME, Gomes EN, et al. Produção e qualidade de mel na Zona da Mata de Pernambuco. 2017;14(26):p.539 https://doi.org/10.18677/EnciBio_2017B52

27. Ministério da Saúde (BR). Fundação Nacional de Saúde. Manual de diagnóstico e tratamento de acidentes por animais peçonhentos. Brasília. 2001;(2):120p. Disponível em: https://www.icict.fiocruz.br/sites/www.icict.fiocru z.br/files/Manual-de-Diagnostico-e-Tratamentode-Acidentes-por-Animais-Pe--onhentos.pdf 28. Magalhães CS, Balta SLSMA, Bezerra MLMB, et al. Aspectos epidemiológicos e clínicos dos acidentes ofídicos ocorridos nos estados de Alagoas e Pernambuco. RESMA. 2020;10:119132. Disponível em: https://desafioonline.ufms.br/index.php/sameamb/ article/view/9953

29. Pinto HA, Oliveira FP, Santana JSS, et al. Programa Mais Médicos: avaliando a implantação do eixo provimento de 2013 a 2015. Interface. 2017;21(Supl.1):1087-101. http://dx.doi.org/10.1590/1807-57622016.0520 30. Ministério da Saúde (BR). Secretaria de Vigilância em Saúde. Vigilância em saúde no Brasil 2003|2019: da criação da Secretaria de Vigilância em Saúde aos dias atuais. Bol Epidemiol. 2019;50(n.esp.):156p. Disponível em: https://portalarquivos2.saude.gov.br/images/pdf/2 019/setembro/25/boletim-especial-21ago19web.pdf?fbclid=IwAR3qTQYyo5tG7dYLNXWfj 4ymtmIAoJtlolbTnwToAPConwrDn0Vpv0kyzvo 31. Oliveira SS, Cruz JVF, Silva MA. Perfil epidemiológico de escorpionismo no Nordeste brasileiro (2009 a 2019). Brazilian Journal of Development, 2021;7(2):11984-11996. https://doi.org/10.34117/bjdv7n2-022

32. Faria G, Lima AAM. Aspectos epidemiológicos dos acidentes por animais peçonhentos ocorridos no município de CacoalRO, no período de 2007-2016. RAS. 2019;17(61):45-56.

https://doi.org/10.13037/ras.vol17n61.5867

33. Ministério da Saúde (BR). Sistema de Informação de Agravos de Notificação. Acidente por Animais Peçonhentos - Instrucional de preenchimento da ficha de notificação/investigação. Disponível em: http://portalsinan.saude.gov.br/images/documento s/Agravos/AAP/Animais_Peconhentos_v5_isntr.p df 